\title{
Application of Fast Fourier Transforms in Some Advanced Electroanalytical Methods
}

\author{
Parviz Norouzi ${ }^{1}$, Morteza Pirali-Hamedani ${ }^{2,3}$, Tayebeh Mirzaei Garakani ${ }^{1}$ \\ and Mohammad Reza Ganjali ${ }^{1}$ \\ ${ }^{1}$ Center of Excellence in Electrochemistry, Faculty of Chemistry, \\ University of Tehran, Tehran, \\ ${ }^{2}$ Department of Medical Chemistry, Faculty of Pharmacy, \\ Tehran University of Medical Sciences, Tehran, \\ ${ }^{3}$ Pharmaceutical Sciences Research Center, Tehran, \\ Iran
}

\section{Introduction}

In the most of electrochemical (EC) experiments, measurements mostly are performed in the time domain. However, in some cases, we require more information for the obtained data such as knowledge about the frequency content and behavior of the electroanalytical signals and of complete systems. Fortunately, there exists a defined method for transforming data from the time domain into the frequency domain, where information exist about the spectral content of EC data. The method for this propos is Fourier Transform (FT), which has the ability to convert a time domain data to the complex frequency domain, meaning the spectral data contains information about both the amplitude and phase of the sinusoidal components that make up the signal. In addition, the inverse FT, converts the generated complex frequency-domain signal data back into the time-domain without losing wanted information. Accordingly, it can say that both the time- and frequency-domain data complement and the two domains can provide a different view of the same EC data.

Application of fast Fourier transformation (FFT) algorithm for numerical EC data provides the complex spectrum according to magnitude and phase, which can be used for real time analysis. In this direction, in modern electrochemistry, FFT has been used for digital signal processing and filtering. Also, the FFT process returns a vector of real and imaginary elements, which represent the various resolved harmonics in impedance spectroscopy, AC and square wave voltammetry (Popkirov, 1996).

On the other hand, it must be noted that in all EC data collection, to hold on the sampling theorem for FFT, the bandwidth of the input signal is limited by an analog low pass filter (cutoff frequency $f_{c}=f_{i n, \max }$ ) ahead of the Analog to Digital (A/D) converter. In fact, after collecting data in the computer memory, they are used for calculating the signal in the frequency domain.

This chapter serves as summary application of the FFT analysis techniques implemented in EC measurement platform. By reading through this document, you will receive a comprehension of the fundamental concepts in FFT-based measurements used throughout EC application, providing you insights to better understanding of the measurement 
parameters, procedures, and resulting EC data. In addition, this chapter describes the general operation of the FFT analysis accompanied with modern EC methods.

\section{Basic FFT theory}

The FT, a pervasive and adaptable tool, is used in many fields of science as a mathematical technique to alter a problem into one that can more easily be solved. Scientists consider FT theory as a physical phenomenon, not simply as a mathematical tool. Based on the Fourier theory, any signal in periodic manner in the time domain can be derived from the sum of sine and cosine signals of different frequencies and amplitudes, which is called as a Fourier series (Weaver, 1983). Thereby, it is notable to calculate the frequency spectrum of a periodic signal according to Equation 1,

$$
x(t)=\frac{A_{0}}{2}+\sum_{n=1}^{\infty} A_{n} \cdot \sin \left(n \cdot \omega_{0} \cdot t\right)+\sum_{n=1}^{\infty} B_{n} \cdot \cos \left(n \cdot \omega_{0} \cdot t\right)
$$

where, $x(t)$ is data in time domain, $A_{n}$ and $B_{n}$ are the amplitude, $w_{o}$ is the frequency of the waveform and $\mathrm{n}$ is the harmonic number. Each of these elements leads to a discrete component in the frequency domain, and periodic signals exhibit discrete line spectra. However, signals with a non-periodic characteristic in the time domain cannot be described by FT, and those signals exhibit a continuous frequency spectrum with a frequencydependency. Therefore, the frequency spectrum of such signals is not composed of discrete spectral components. The signal in the frequency domain is calculated by means of a FT (Equation 2).

$$
X_{f}(f)=F(x(t))=\int_{-\infty}^{\infty} x(t) e^{-j 2 \pi f t} d t
$$

Also, for measuring the harmonic section of the EC data, it is more useful to examine the signal in the frequency domain (Rauscher et al., 2001). It has been shown that the signal in the frequency domain of the fundamental (1st order harmonic) is superimposed by several higher-order harmonics with the aid of a spectrum analyzer. As a matter of fact, this information cannot be simply obtained by examining the signal in the time domain. Practically, the higher order harmonics are not possible, and limited number of the data samples can be used for FFT calculation.

\section{Fundamentals of electroanalytical signals}

It is well known that many fundamental microscopic processes take place on the electrode surface, which can lead to the overall electrical signals. They may include the transport of electrons through the electronic conductors, the transfer of electrons at the electrode/electrolyte interfaces to form species which are originated from the cell materials, and also, the stream of charged atoms. Indeed, the current depends on the ohmic resistance of the electrodes and the electrolyte and also on the process rates at the electrode/electrolyte interfaces.

In practical point of view, there are three different types of electrical signals in EC measurements. Each basic electrical measurement of current $(i)$, resistance $(R)$, and potential $(V)$ has been used alone or in combination for analytical measurements (Brett \& Brett, 1993). 
First, in transient measurements a waveform function of potential may be applied at electrode surface and then the resulting time varying current measured. The ratio voltage to current often called the time varying resistance, measures the impedance resulting from the voltage perturbation at the electrode/solution interfaces. If a FFT is used, a distortion arises because of the non-periodicity of excitation. Such transformation is only valid when the applied potential waveform is sufficiently small so that system response becomes linear .

The second type contains signals containing random noise, and measure the resulting current and voltage, and application of FFT to the results to obtain the frequency domain data. This process can be used in electrochemical noise analysis (ENA) method for determination of corrosion. This approach offers the advantage of fast data collection because only one signal is applied to the interface for a short time.

The last type, the most common and standard one, is to measure EC data by applying a single-frequency voltage or current to the electrode interface and measuring the phase shift and amplitude, which leads to measure the real and imaginary parts of the resulting current at a certain frequency. The most important advantage of such FFT analysis is in AC and Square Wave Voltammetry (SWV), in which combines the first and third techniques.

\section{Application of FFT in electroanalytical methods}

As mentioned above, most electrical signals in EC measurements may be examined in the time domain with the aid of a potentiostat and in the frequency domain with the aid of the computer digital spectrum analyzer. The two display modes are related to each other, where each signal variable in the time domain has a frequency spectrum characteristic (Gavaghan \& Bond, 2000). This calculation would be obtained in a continuous data collection, so the frequency resolution would be unlimited. Noticeably such exact calculations are not possible in practice, where by given certain prerequisites, the spectrum can be determined with sufficient accuracy. In practice, the FT data is constructed with the aid of digital signal processing, as a result, the signal to be analyzed has to be sampled by an A/D converter and quantized in amplitude.

Most of the generated electrical signals in electroanalytical methods are continuous, in which for every time value there is a defined data. However, in order these continuous signals to be analyzed, it is needed a computer-based measurement system employed, such as Labveiw interfacing program or other interfacing system. Those systems can convert the EC electrical signal into a stream of digital data, which each data represents a numeric value that is proportional to the measured data at a specific time. This process is known as data sampling: converting the analog signals into a discrete-time signal (a process handled by A/D converter) to allow electroanalytical data in a wide level range to be simultaneously processed by FFT program and to be displayed on the computer screen (Norouzi et al., 2003).

In the computing process, the FFT method operates by decomposing $N$ data point in time domain signal into $N$ frequency domain signals each composed of a single point of the electroanalytical measurement. The next step is to calculate the $N$ frequency spectra corresponding to these $N$ time domain signals. Finally, the $N$ spectra are synthesized into a single frequency spectrum. If $\mathrm{N}$ is an integer power of 2 , thus $\mathrm{N}=2^{p} \quad(\mathrm{p}=1,2,3, \ldots)$, use the fastest FT function that uses the decimation-in-time algorithm. Given N samples of a periodic function $f(t)$ by a normalized sampling rate $(T=1),\{f(0), f(1), f(2), \ldots . f(N-1)\}$, the discrete Fourier transform (DFT) is defined by: 


$$
F(k)=F_{r}(k)+i \cdot F_{i}(k)=\sum_{n=0}^{N-1} f(n) \cdot[\cos (2 \pi n k / N)-i \cdot \sin (2 \pi n k / N)]
$$

Normally, at first, an electrode response was recorded and then, DFT was applied on the collected data and the existing frequencies, phase angle, real and imaginary parts are calculated. Based on this calculation, the modern methods are established, such as ENA, FFT Cyclic voltammetry, FFT SW voltammetry and FFT impedance spectroscopy.

\subsection{Application of FFT electrochemical measurements based on noise analysis}

The frequencies of unwanted signals in EC data, with noise or random characteristics, cannot be found easily (Aballe et al., 1999; Sang et al., 2009; Dai, 2000). The analyst requires a plot of the intensity at each individual frequency in order to make identification and the EC signal to be interpreted. A means of "decoding" for calculating the individual frequencies is needed. This procedure can be accomplished via a well-known mathematical technique such as DFT. This transformation is performed by the computer based programs, which then presents the user the desired spectral information. When the frequency region of the noise is found, it can be used for two aims in the EC analysis; data filtering for enhancing signal-to-noise (S/N), and corrosion monitoring (Darowicki \& Zieliski, 2001; Safizadeh\& Ghali, 2010).

\subsection{Application of FFT in cyclic potential sweep voltammetry based on filtering}

In modern EC methods, the FFT analysis voltammetry were developed in order to overcome some existing limitations encountered with electronic instrumentations. In fact, the selectivity and percision characteristics of the classic electroanalytical methods depend on the number of filter circuits. Normally, the most potentiostat typically has many filter circuits that are arranged before and after the amplifier. The main problem here is to apply a fast excitation signal for fast EC measurement. Actually, application a fast excitation signal can produce a large charging current due to existing capacitor in the analog filters.

On the other hand, by using FFT filtering method, instead of the analog filters, the signal can be measured very quickly. Consequently, the time element per sample is reduced to a matter of less than second $\left(10^{-9} \mathrm{~s}\right)$ rather than several minutes which happened in the classical analog measurements. As cyclic voltammetry finds its greatest use in the study of fast electrochemical process, and transient intermediates, we are supposed to choose this method for its description and the FFT application. Through the use of analog filters, the maximum permissible sweep speed is limited by the transient time of the applied filter. The maximum span that can be analyzed at a specific resolution by means of FFT is limited by the sampling rate of the A/D converter and by the memory available for saving the sampled data. In fact, to allow shorter sweep times, FFT digital filters are advantageous for narrow resolution bandwidths. Basically, the EC digital analyzer is designed for bandwidths from $100 \mathrm{~Hz}$ to $10 \mathrm{MHz}$. This digital filtration provides condition that can be used for very high potential scan rates on working electrode for electrochemical measurement in flowing solution.

Figure 1 shows an example for FFT filtration for fast cyclic voltammetric measurement (Norouzi et al. 2007). In this method at the beginning, a CV of the electrode was recorded (see Fig. 1a) and then by applying FFT on the collected data, the existing high frequency noises were indicated (see Fig. 1b). Finally, by using this information, the cutoff frequency of 
the analog filter was set at a certain value (where the noises were removed from the CV). The resulted CV in Fig. 1c shows successfulness of the filtering procedure. This kind of filtration and also current integration significantly reduces the noise level in the obtained data.

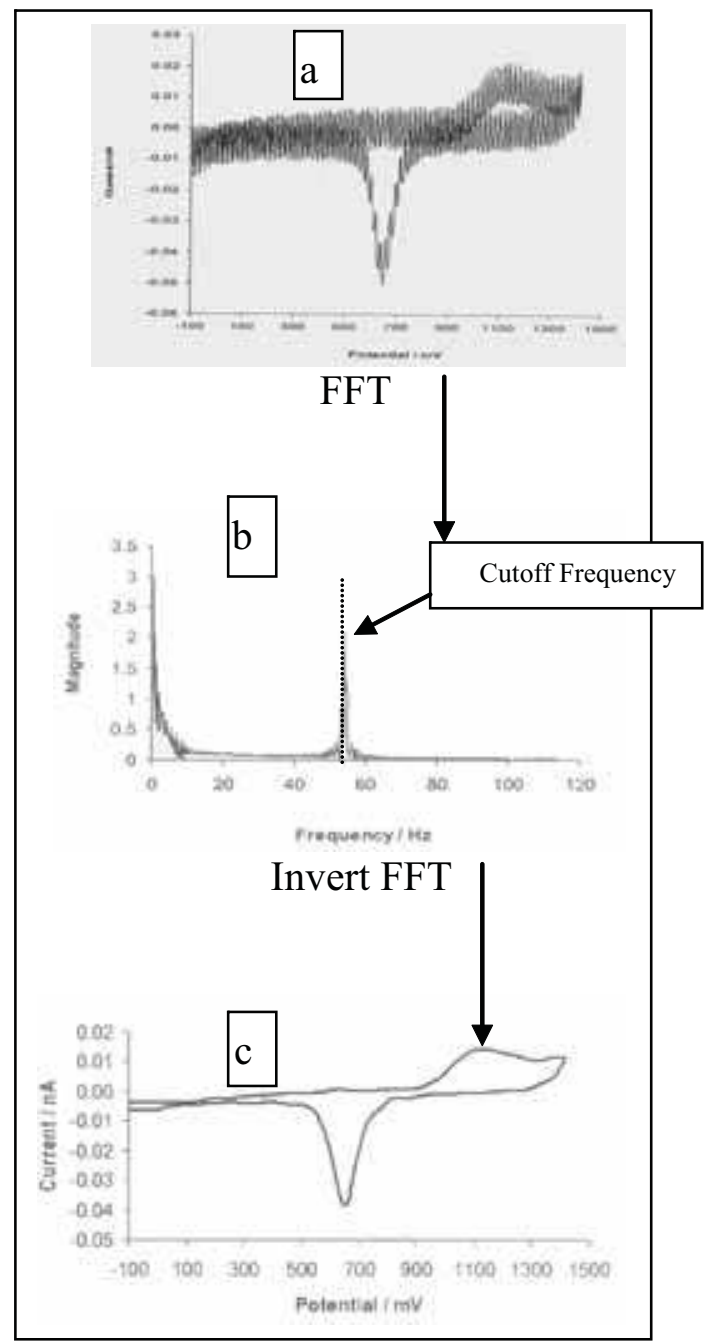

Fig. 1. Application of FFT filtration to smooth a noisy CV, a) original CV, b) FFT spectrum of the $\mathrm{CV}$ (the inset shows the cutoff frequency that is selected for filtration), c) the resulted CV after removing the noise frequencies

The EC measurement based on the FFTdigital filters is widely used by Norouzi group for determination of several organic compounds and drugs such as; Diphenhydramine (Norouzi et al. 2010 b), Lidocaine (Norouzi at al. 2007), Methyldopa (Norouzi et al. 2009), and Salbutamol(Ganjali et al. 2005). The EC method under reported condition was named FFT Continuous Cyclic Voltammetry (FFTCCV). In this method the potential waveform was 
continuously applied during an experiment run, where the collected data was digitally filtered by the technique, before using them in calculation for the analyte response (Norouzi et al., 2010).

It must be noted that, this digital analyzing, also, offers the possibility of the frequencydependent gain. Recorded spectra can automatically be displayed with the correct levels. In these cases a reduction of the displayed noise by decreasing the resolution bandwidth is not permitted. Due to this fact that, the sensitivity is also important for the fast measurement speeds the program of the FFT digital analyzer featuring a low noise figure leads to the use of greater resolution bandwidths, and also with manual setting of the resolution and bandwidths, the sweep time can be adapted automatically.

An example of application of that waveform is shown in Figure 2. This figure shows a sequence of CVs recorded during the flow injection of $50 \mu \mathrm{L}$ of $1.0 \times 10^{-6} \mathrm{M} \mathrm{Cl}^{-}$(in $0.05 \mathrm{M}$ $\mathrm{H}_{3} \mathrm{PO}_{4}$ ) into the eluent solution containing $0.05 \mathrm{M} \mathrm{H}_{3} \mathrm{PO}_{4}$. The potential axis on this graph represents potential applied to the working electrode during each sweep. The time axis represents the time passing between the beginning of the flow injection experiment and the beginning of a particular sweep (i.e. it represents a quantity proportional to the sweep number). The characteristic element of CVs at gold electrode is a set of peaks associated with the formation and dissolution of a surface oxide layer at about 1600 and $400 \mathrm{mV}$ (when potential sweep rate is $\left.20 \mathrm{Vs}^{-1}\right)$, respectively. The process is also initiated by the electrosorption of the hydroxyl ion, which at more positive potentials undergoes deprotonation and structural rearrangement. The surface oxidation can be initiated by adsorption of water molecule and then at more positive potential $\mathrm{AuOH}$ forms leading to the formation of a two-dimensional phase of gold oxide;

$$
\mathrm{Au}\left(\mathrm{H}_{2} \mathrm{O}\right) \rightarrow \mathrm{AuOH}+\mathrm{e}+\mathrm{H}^{+}
$$

At more positive potentials we have $\mathrm{AuO}$ according to the following reaction;

$$
\mathrm{AuOH} \rightarrow \mathrm{AuO}+\mathrm{e}+\mathrm{H}^{+}
$$

Figure $2 \mathrm{~b}$ shows the absolute current changes in the CVs curves after subtracting the average background of $10 \mathrm{CVs}$ (in the absence of the analyte). As can be seen, this way of presentation of the electrode response gives more details about the effect of adsorbed ion on currents of the CV. The curves show that current changes mainly take place at the potential regions of the oxidation and reduction of gold. When the electrode-solution interface is exposed to $\mathrm{Cl}^{-}$, which can adsorb on the electrode surface, the oxide formation process is inhibited.

All CV curves observed during the entire experiment (typically 100 to 1000 curves) were always stored in computer memory and could be saved onto a hard drive for future analysis (See Fig. 2a). The important point in FFTCCV method is that parts of the adsorbed analyte still remain on the electrode surface that can inhibit the oxidation process of the electrode surface. In this method, $\Delta \mathrm{Q}$ is calculated based on the all-current changes at the CV (See Fig. 2c). A total absolute difference function can be calculated by using the following equation:

$$
\Delta Q_{T A}(s \tau)=\Delta t\left[\sum_{E=E_{i}}^{E=E_{v}}\left|i(s, E)-i\left(s_{r}, E\right)\right|+\sum_{E=E_{v}}^{E=E_{i}}\left|i(s, E)-i\left(s_{r}, E\right)\right|\right]
$$



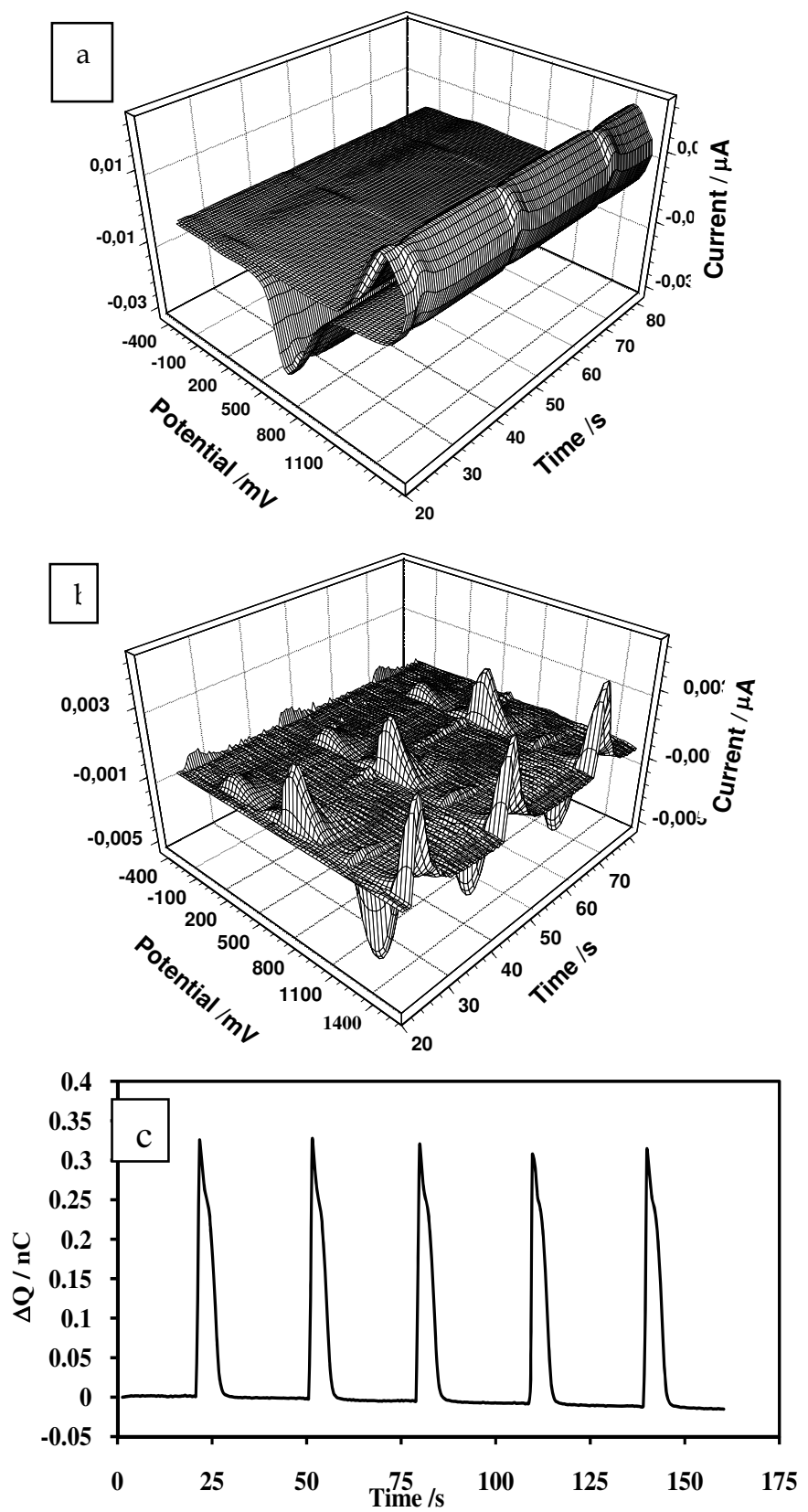

Fig. 2. a) Cyclic voltammograms at Au ultra-microelectrode recorded during the flow injection. The eluent was $0.05 \mathrm{M} \mathrm{H}_{3} \mathrm{PO}_{4}$ with the flow rate of $0.5 \mathrm{~mL} / \mathrm{min}$., b) Curves resulted from subtracting the CVs in fig. a, from the average of $10 \mathrm{CVs}$ (in the absence of analyte), c) Response of $\mathrm{Au}$ ultramicroelectrode to 5 consecutive injections of analyte 
Where, $\mathrm{s}$ is the sweep number, $\tau$ is the time period between subsequent sweeps, $\Delta t$ is the time difference between two subsequent points on the CV curves, i $(s, E)$ represents the current of the CV curve recorded during the s-th sweep and i $\left(s_{r}, E\right)$ is the reference current of the $C V$ curve. $E_{i}$ and $E_{0}$ are the initial and the vertex potential, respectively. The reference $\mathrm{CV}$ curve was obtained by averaging a few $\mathrm{CV}$ curves recorded at the beginning of the experiment (i.e. before injection of the analyte). These equations show that for the same flow injection experiment the analyte response can be obtained using different integration limits.

It should be noted that in this method, all studied processes involve adsorption of analyte; hence both charging and faradic currents may potentially carry useful analytical information. To get such information, it was important to sample current at a frequency at least two times higher than the current transducer bandwidth. In order to fulfill this requirement the sampling frequency was always adjusted at $100 \mathrm{kHz}$. In addition FFT digital low pass filters with $0.5-30 \mathrm{kHz}$ cutoff frequencies lead to remove noise from the data. If the main contribution to the baseline noise is from the "white" noise generated by the potentiostat, the integration procedure usually provides a 3 to 60 fold improvement in $\mathrm{S} / \mathrm{N}$ compared to the simple monitoring of the current at a fixed potential. However, in the case of severe environmental noise (e.g. power line noise) the improvement may be much larger. Signals with weak level are thus shown more distinctly in the voltammogram and the measured level values are thereby stabilized and reproducible. In the case of a sinusoidal signal, the displayed level is not influenced by a reduction of the bandwidth. To obtain stable and reproducible results of noise measurements, a narrow bandwidth should be selected. The noise bandwidth is thus reduced and high noise peaks are better to be averaged.

\section{Application of FFT in electrochemical noise analysis}

As mentioned above the identification of noise frequencies in the electrochemical data by FFT method, can be used for study of some processes that occur on the electrode surface. This type of noises, which are created by EC process, called electrochemical noise (EN). For many years, EN has been observed during corrosion and other electrochemical reactions, and the phenomenon is well established.

The theoretical behavior of EN is not, completely, discussed, but there have been many useful applications, both in scientific surface and in corrosion monitoring studies. In an EC system, noise of potential and of current can be made independently or together during corrosion reaction that can be take placed on the electrode surface. The important point is that EN measurement technique does not involve any external perturbation of the corroding system. In fact, the instruments required to perform EN measurements are reasonably simple, particularly with FFT digital analyzer and modern computer-based data acquisition techniques. More commonly, the EN curve for potential and current has an appearance similar to that shown in Figure 3.

Generally, localized corrosion processes tend to give particularly strong EN signals, which consist of low frequency $(<1 \mathrm{~Hz})$ and small amplitude signals. Those signals are spontaneously generated by electrochemical reactions occurring at corroding or other surfaces (Zaveri et al., 2007). However, In this process, both the noise of potential and current are of the $1 / f$ type. The range of frequencies depends on the sampling interval $\Delta t$ (typically $0.5 \mathrm{~s}$ ) and on the number of readings $M$ of a time record. The maximum and 
minimum frequencies that can be analyzed are: $f_{\max }=1 / 2 \Delta t$ and $f_{\min }=1 / M t$ ). The limiting value at a frequency approaching zero is defined as the spectral noise resistance.

In practice, spectral noise plots can be obtained only in a frequency range that is more limited than in EIS. On the high frequency side, the limit is imposed by the instrumental noise, whereas in the low frequency region, the time of acquisition becomes very long (Lafront et al., 2010).

In a different approach, the time record of potential or current is converted into a power spectral density (PSD), which is the distribution of the power in the frequency domain. This transformation is usually made by means of the FFT method. Figure 4 shows the calculated power spectral density (PSD $=1 / f n$, where $n$ is a constant). The figure shows that PSD falls with increasing frequency, giving a straight line on a log-log plot, implying a relationship. In addition to the sloping $1 / f r$ region of the spectrum, there are often indications of plateaus at the high and/or low frequency ends of the spectrum. Potential noise frequency relation changes have been noticed in the form of a component of character $1 / f 2$, this being attributed to pit initiation.

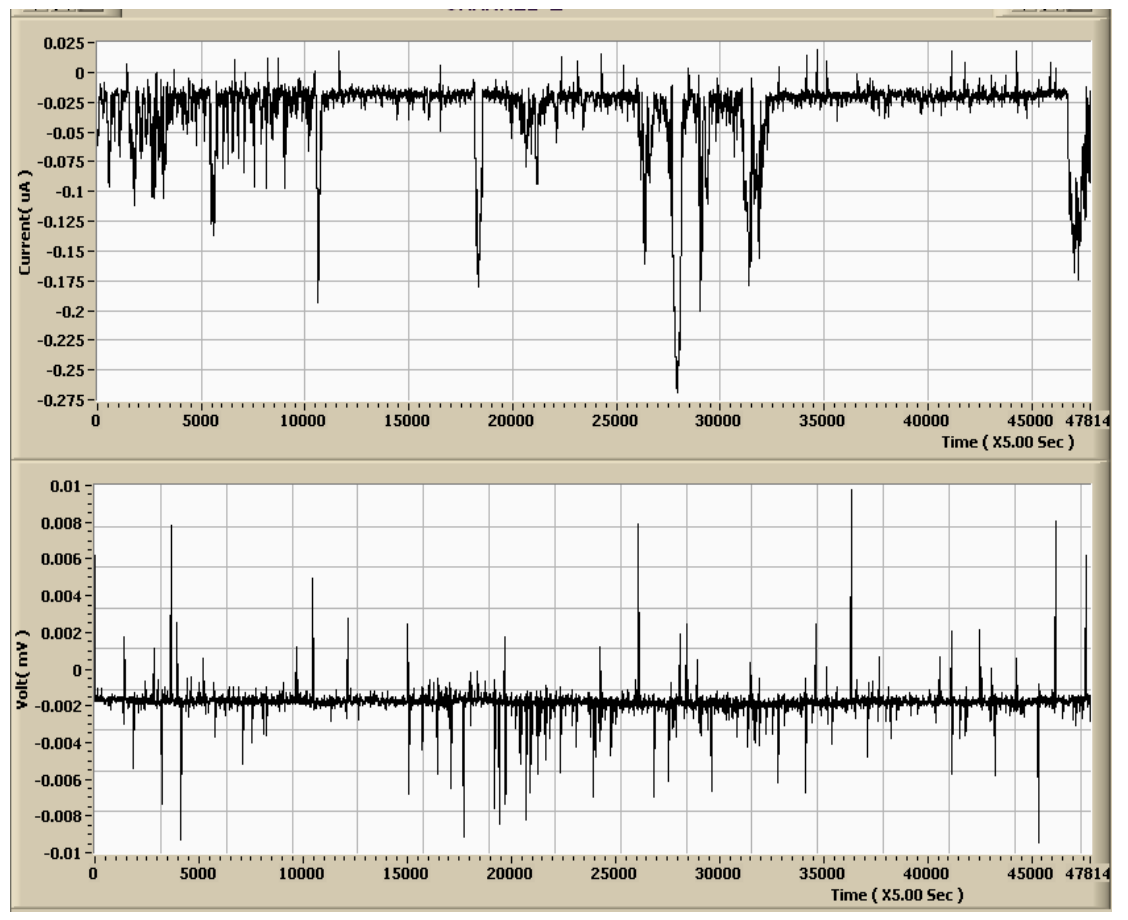

Fig. 3. The sample graph for $\mathrm{EN}$ measurements of an $\mathrm{Al}$ palate in $\mathrm{NaCl} 0.01 \mathrm{M}$, top) Current noise, bottom) Potential noise

The character of PSD changes as a function of frequency depend on the type of corrosion and has also been the subject of investigations. The area under the curve is the total power in the signal, and is identical to the standard deviation calculated from the time record. Thus, as the frequency spectrum moves to higher PSDs, so the rate of reaction may be expected to increase. The details of the calculation are beyond the scope of this book. 


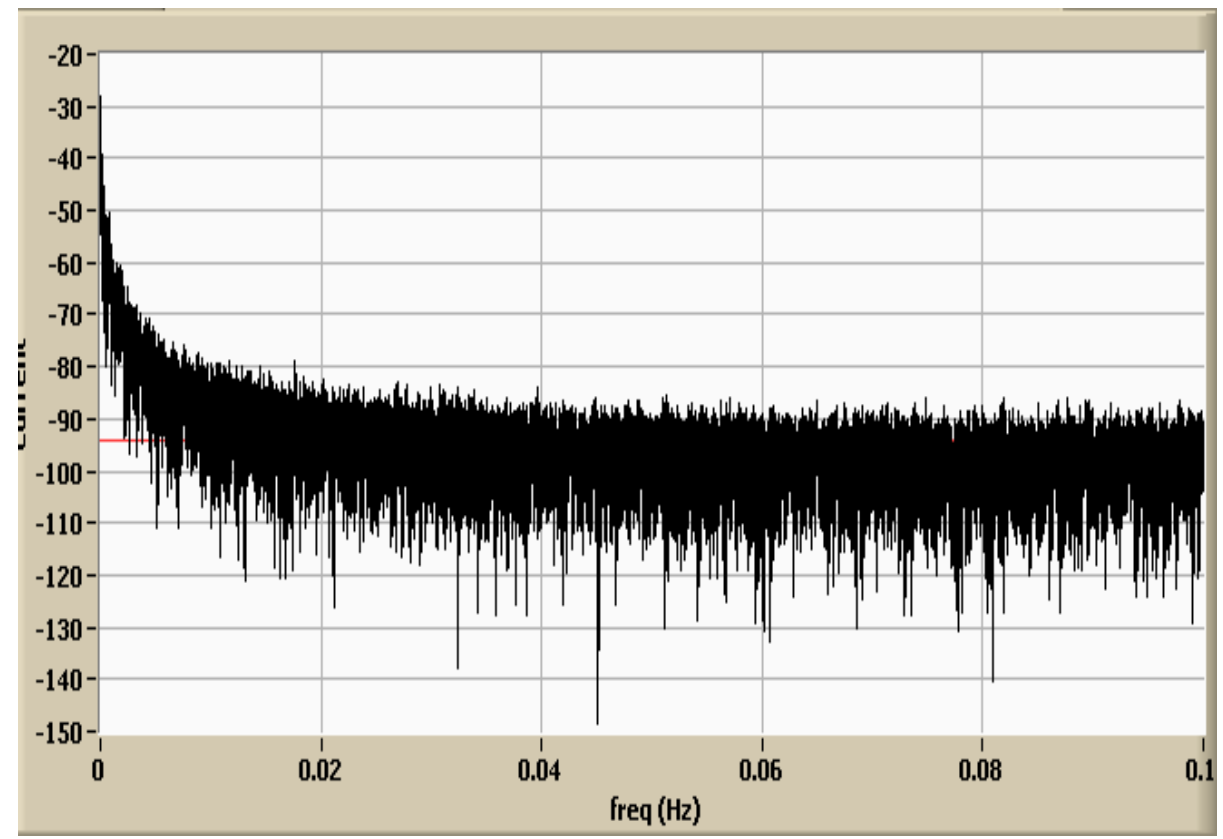

Fig. 4. The sample of PSD graph for EN measurements of an $\mathrm{Al}$ plate in $\mathrm{NaCl} 0.01 \mathrm{M}$

\section{Application of FFT in AC voltammetry}

The determination of the characteristics of the EC data system by AC techniques requires measuring the impedance at various frequencies, to result a frequency spectrum (Bond et al., 1997). The most dominant application of FFT methods in AC voltammetry is in calculation of the impedance of the electrode response, which is known as electrochemical impedance spectroscopy (EIS).

By definition, AC voltammetry (ACV) utilizes a small-amplitude sine wave which is added to a potential ramp to modulate the current output. In fact, $\mathrm{ACV}$ is an extension of classical linear sweep techniques such as cyclic voltammetry. A DC ramp with a comparatively slow sweep rate and an AC signal are superimposed and applied to a working electrode, and the response of the $\mathrm{AC}$ current and its phase angle are registered. In general, modulation potential amplitude up to $20 \mathrm{mV}$ is used; higher amplitudes are not used to specifically avoid contributions from higher order harmonics. This may be considered as a limiting case for ACV. In fact, all of the information is intentionally contained in only the lowest order harmonics. Its main strength lies in the quantitative characterization of electrode processes, and it can also be used for analytical purposes.

A second general approach has employed AC voltammetry and the mathematics of the FFT. This technique was introduced and used extensively by (Smith, 1976). In it, the perturbation signal is composed of a sum of selected sinusoids. This approach, although powerful, is quite expensive and complex as well, and has not been widely employed. Both experimental implementation and data analysis employ a small-amplitude excitation waveform. 
The applied excitation waveform consists of a fundamental harmonic frequency $f_{0}$ and a number of odd harmonics $(2 n+1) f_{0}$. This arrangement is superior to other excitation waveforms. All these frequencies are applied at the same time and the data to each frequency is found by the FFT. Depending on the method used for the data acquisition, different methods are used for data validation. In case of a sinusoidal signal were applied to a nonlinear system, the data function would contain multiples harmonics of the excitation waveforms.

The techniques commonly employed for AC-impedance measurements in modern equipment can be subdivided into two main groups: single-sine and multiple-sine methods. The lock-in technique and frequency-response analysis will be described as representatives of the single sine techniques and FFTs will be introduced as an example for multiple-sine techniques. In single-sine methods, a small-amplitude sinusoidal signal with a fixed frequency is applied to the test cell. The response signal is then analyzed to extract the two components of the impedance (real and imaginary parts or magnitude and phase).

Here, invalid impedance data cannot only be caused by nonlinearity but very frequently by a lack of stability of the system under investigation. Impedance test equipment usually comprises an AC measurement unit and a potentiostat or galvanostat. For many applications, such as biomedical investigations or the characterization of thin films in which it is not essential to maintain a DC-voltage level during the impedance measurement, a potentiostat is not required (Házì et al., 1997).

EIS is frequently used to characterize systems that are changed during the time. Another way to reduce the effects of a system changing during the measurement is to reduce the total measurement time by using a multi-sine technique, which is FFT method. Figure 6 shows typical curve obtained by this technique for electrodeposition of $\mathrm{Cd}$ on a gold electrode in $0.1 \mathrm{M} \mathrm{Cd} \mathrm{SO}_{4}$.

In the case of multi-sine techniques, a measurement is carried out at several frequencies simultaneously. The phases of the superimposed signals are randomized to minimize the amplitude of the composite signal (see Fig. 5b). In contrast to single-sine techniques, multisine techniques do not require waiting for a full cycle to be completed for each of the frequencies used. The time-domain signals are digitized and transferred into the frequency domain by carrying out the FFT. The resulting data for each discrete frequency can be treated the same way as the impedance data obtained with a single sine technique. Repeated application of the waveform and averaging of the signal before FFT is applied can improve the $\mathrm{S} / \mathrm{N}$ ratio of the multi-sine technique, although it increases the measurement time required. The impedance data obtained in this manner can be presented in several different formats.

Impedance spectra $Z_{\text {real }}$ versus $Z_{\text {img }}$ measured at a number of fixed DC potentials are suitable for quantitative studies of redox reaction kinetics whereas potential-dependent admittance values $(1 / Z)$ obtained at fixed frequencies can provide AC voltammograms that are more readily interpreted in electroanalysis. The ratio of the two is the impedance of the test electrode. The measurement is then repeated at another frequency. The impedance spectra measured in this work were usually shaped as shown in the following Nyquist- and Bode-plot. Once real and imaginary parts of the input signals have been determined by correlation, the complex impedance of the test object can be calculated. It can be mathematically proven that all the spurious components are rejected by this technique of correlation provided that a sufficiently large number of cycles have been used for the integration (Garland et al., 2002). 

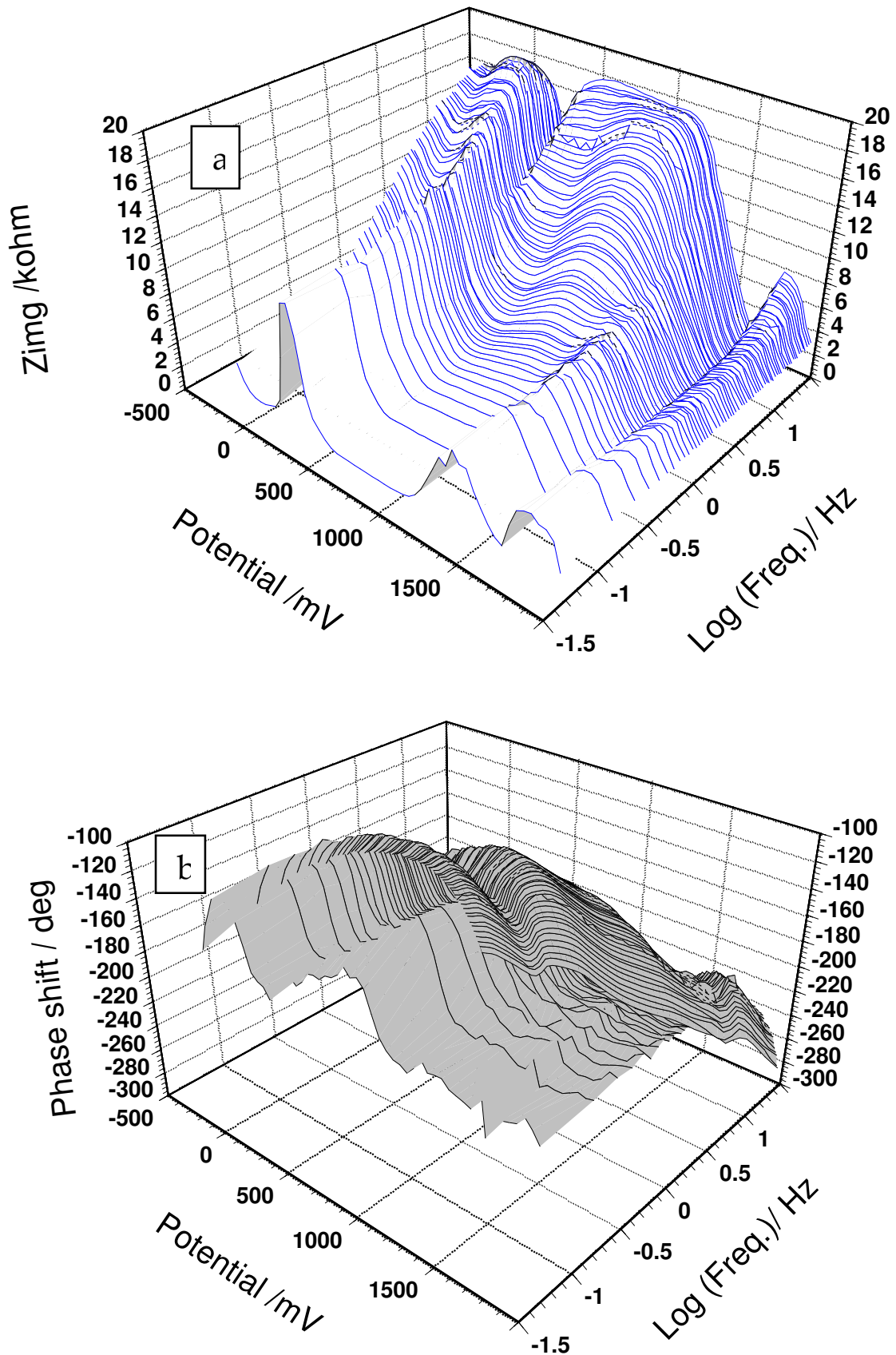

Fig. 5. Typical FFT EIS graph for electrodeposition of Cd on gold electrode, a) Z imaginary, b) phase shift, changes in deterrent frequency and potential 
The advantage of modern FFT technique is that the information is obtained quickly; therefore it may be used to study impedances evolving with time. The limitation of the FFT technique is that the response to individual frequencies is usually weaker than that when only one frequency is used. It should be added that other types of analysis of system responses were also used, for example, Laplace transform of the applied perturbation and the response to determine the impedance spectra (Carstensen et al., 2008). The time-domain signals are digitized and transferred into the frequency domain by carrying out a FFT. The resulting data for each discrete frequency can be treated the same way as the impedance data obtained with a single sine technique.

In modern EIS analysis, lower frequency data are usually measured in the time domain. The current response is then measured using an A/D computer. In this case the FFT is used to convert the current signal into the frequency domain as carried out for other techniques. The FFT capabilities have subsequently been incorporated into several commercial instruments, primarily to speed up the acquisition of impedance data at low frequencies by exploiting the multiplex character of the technique. Such determinations are normally carried out at a single, fixed DC potential. In order to obtain potential-dependent impedance data, repeated experiments at different applied DC potentials are therefore required (Arundell et al., 2004).

The use of the FFT in combination with a controlled sequence of potential steps or pulses has been shown to offer an approach by which these time-consuming repetitions can be avoided and impedance measurements can be collected over a wide range of frequencies and DC potentials in a single experiment. However, here, the voltammetric waveforms composed of a sequence of potential steps are ideally suited in mathematical modeling based on the techniques of numerical integration. This approach is elegant in its generality, can be made arbitrarily precise, and is extremely efficient (Baranski et al., 1996). Baranski developed a technique in which a small amplitude square-wave potential perturbation is superimposed upon a potential staircase and in which the FFT is employed to convert current measurements taken as a function of time during several cycles of the square wave at each step potential to the frequency domain. Higher harmonics can be detected by FRAs or lock-in amplifiers, which can be tuned to detect a multiple of the excitation frequency. An alternative is to extract the harmonic signals from the response using FFT.

\section{Application of FFT in SWV}

The idea of obtaining electrode admittance from transient current time curves was investigated previously by Pilla (Pilla, 1972). The speed and sensitivity of SWV is its main advantage (Osteryoung \& O'Dea, 1986). In the last years, data acquisition boards in the electroanalytical instrumentation have improved significantly for carrying out SW voltammetric analysis. The SWV has been used for study of the kinetics of electrode process (Winston et al., 1988). Nevertheless such applications of this method are relatively rare, which may be as a result of the rather complicated equations relating the current response of the electrode to the kinetic parameters of the electrode processes. Also, the theory of SWV does not take into account the effect of uncompensated solution resistance and a distortion of the EC signal by a slow response of a current transducer. These problems are not easy to be solved in any time domain voltammetric techniques. Because the electrode response under AC voltammetric conditions is represented as an admittance (i.e. in the frequency 
domain), all data manipulations needed for obtaining kinetic information are relatively simple.

The principles of this technique are simple. Normally, FFTSWV experiments are done under conditions identical to the traditional SWV, but the electrode response at each DC potential is converted into the frequency domain via FFT. Therefore, FFTSWV measures the admittance of the electrode as a function of potential (Baranski \& Szulborska, 1994). The resultant data are almost similar to those obtained under AC voltammetric conditions.

Indeed, in comparison with traditional AC voltammetry the equipment is much simpler and less expensive, measurements are carried out much faster and it is possible to obtain information about the admittance of the electrode at different frequencies from a single run.

The potential waveform used in the FFTSW voltammetric measurement consists of many SW pulses were superimposed on a staircase potential function, which was changed by a small potential step of $\Delta \mathrm{E}$ (Norouzi et al., 2008). The values of potential pulse of $S W\left(E_{S W}\right)$ and $\Delta \mathrm{E}$ were in a range of few $\mathrm{mV}(10$ to $50 \mathrm{mV})$. In the computer program, the number of SW cycles, Nc, in each staircase potential step was calculated based on the SW frequency as follows, $\mathrm{Nc}=f_{0} / 400 \mathrm{~Hz}$, for $f_{0}>1400 \mathrm{~Hz}$, and $\mathrm{Nc}=1$ for $f_{0} \leq 1400 \mathrm{~Hz}$. The values of $\mathrm{Nc}, f_{\mathrm{o}}, \mathrm{E}_{\mathrm{sw}}$, $E_{\text {initial }}$ and $E_{\text {vertex }}$ were the variable parameters of the technique, which were optimized for achieving to best detector performance. It should be noted that in this method all processes studied involve adsorption of analytes hence both charging and faradic currents may potentially carry useful analytical information.

To get such information, it was important to sample data current at a frequency at least two times higher than the current transducer bandwidth. In order to fulfill this requirement the data sampling frequency was always adjusted between 50 and $100 \mathrm{kHz}$ (depending on scan rate). In addition a second order low pass filter with a $20 \mathrm{kHz}$ cutoff frequency was placed between the current output of the potentiostat and the data acquisition board. In the computer program, the discrete FFT analysis was used for data processing. If one SW cycle per potential step is applied, the time domain response resulted with this method is similar to that which obtained using Osteryoung SW voltammetry. Here either four data points per SW cycle were collected. If there is more than one cycle at one potential step, the current recorded in different cycles at the same DC potential is averaged (i.e. the first data points in every cycle are added together and divided by the number of cycles then the second and subsequent data points are treated in the same way) (Baranski \& Szulborska, 1994).

The first component in Eq. 5 gives the imaginary part $\left(Z_{\mathrm{img}}\right)$ of the impedance and the second part gives its real component $\left(Z_{\text {rel }}\right)$. A full discussion for the determination of $Z_{\text {rel }}$ and $Z_{\text {im }}$ based on the sampled currents $\left(\mathrm{I}_{\mathrm{s}}\right)$ will be given in the next section. Theoretically, the detector impedance,

$$
|Z|=\sqrt{Z_{i m g}^{2}+Z_{r e l}^{2}}
$$

where $|Z|$ in a specific frequency is equal to $\mathrm{E} / \mathrm{I}$.

Application of discrete FFT analysis on the sampled current requires a specific method in current sampling. The admittance of the electrode is calculated at each potential step by the DFT method. High frequency components are removed by placing an analog low pass filter 
between the current transducer and the A/D converter (Van Valkenburg, 1982). It required the number of sampled currents at each pulse cycle which must be represented by $2^{n}$ (where $n$ is an integer and greater than 1). Therefore the currents, $\mathrm{I}_{\mathrm{s}}$, were sampled at even time intervals, $t_{s,}$

$$
t_{s}=1+\frac{s}{4 f_{0}}
$$

where $s$ is an integer number and changes from 0 to 7 . Therefore if currents are sampled at even time intervals, $\mathrm{t}_{s}, \mathrm{t}_{s+1} / 4 f_{0}, \mathrm{t}_{s+2} / 4 f_{0}$ and $\mathrm{t}_{s+3} / 4 f_{0}$, then the values of the sampled currents will be,

$$
\begin{gathered}
i_{0}=\sum_{n=1} A_{n} \sin \left(2 n \pi f_{0} t_{c}-\phi_{n}\right) \\
i_{1}=\sum_{n=1} A_{n} \sin \left(n \pi / 2+2 n \pi f_{0} t_{s}+\phi_{n}\right) \\
i_{2}=\sum_{n=1} A_{n} \sin \left(n \pi+2 n \pi f_{0} t_{s}+\phi_{n}\right) \\
i_{3}=\sum_{n=1} A_{n} \sin \left(n 3 \pi / 2+2 n \pi f_{0} t_{s}+\phi_{n}\right)
\end{gathered}
$$

The equations show that in the first harmonic $(\mathrm{n}=1)$ the current components $i_{0}$ and $i_{2} \quad$ (as well as, $i_{1}$ and $i_{3}$ ) have a phase shift equal to $\pi$. However, their absolute values are the same with an opposite sign. As mentioned above, the currents were sampled four times per SW cycle, $i_{0}, i_{1}, i_{2}$ and $i_{3}$. In each step, $\Delta \mathrm{E}$, of staircase potential ramp, the total sampled currents were $4 \mathrm{Nc}\left(\mathrm{Nci}_{0}, \mathrm{Nci} i_{1}, \mathrm{Nci}_{2}\right.$ and $\left.\mathrm{Nci} i_{3}\right)$, which were reduced to four by averaging each Nci. Because of dependence of Nc on frequency, at SW frequencies lower than 1400 $\mathrm{Hz}$ lager number of currents were averaged, which could be helpful for reducing the noise level. At the end of each potential ramp, the data were stored in an array matrix as follows,

$$
\text { Data array }=\left\{\begin{array}{cccccccc}
i_{0}^{1} & i_{1}^{1} & i_{2}^{1} & i_{3}^{1} & E_{0} & E_{1} & E_{2} & E_{3} \\
\cdot & \cdot & \cdot & \cdot & \cdot & \cdot & \cdot & \cdot \\
\cdot & \cdot & \cdot & \cdot & \cdot & \cdot & \cdot & \cdot \\
\cdot & \cdot & \cdot & \cdot & \cdot & \cdot & \cdot & \cdot \\
i_{0}^{n} & i_{1}^{n} & i_{2}^{n} & i_{3}^{n} & E_{0} & E_{1} & E_{2} & E_{3}
\end{array}\right\}
$$

where $\mathrm{n}$ is the number of the potential step and $E_{0}$ to $E_{4}$ are the electrode potentials at which the current is sampled.

To calculate the admittance of the detector response, first the real and imaginary components of the alternating current need to be calculated. The real component of $I$ and $\mathrm{E}^{\prime}$ are given by, 


$$
\begin{gathered}
I=i_{2}-i_{0} \\
E^{\prime}=E_{2}-E_{0}=-2 E_{s}
\end{gathered}
$$

and the equation for the imaginary components are,

$$
\begin{gathered}
I^{\prime}=i_{1}-i_{3} \\
E^{\prime \prime}=E_{1}-E_{3}=2 E_{s}
\end{gathered}
$$

Now, the real, $Y^{\prime}$, and imaginary, $Y^{\prime \prime}$, components of the detecting admittance can be calculated as follows,

$$
Y^{\prime}-j Y^{\prime \prime}=\frac{I^{\prime}-j I^{\prime \prime}}{E^{\prime}-j E^{\prime \prime}}=\frac{I^{\prime \prime}-I^{\prime}-j\left(I^{\prime}+I^{\prime \prime}\right)}{4 E_{S}}
$$

and

$$
\begin{gathered}
Y^{\prime}=\frac{i_{0}+i_{1}-i_{2}-i_{3}}{e_{0}+e_{1}-e_{2}-e_{3}} \\
Y^{\prime \prime}=\frac{i_{0}-i_{1}-i_{2}+i_{3}}{e_{0}+e_{1}-e_{2}-e_{3}}
\end{gathered}
$$

Also, the average current, $\mathrm{I}_{s}$ at each potential step is given by

$$
I_{s}=\frac{i_{0}+i_{1}-i_{2}-i_{3}}{4}
$$

The results from application of FFT analysis showed that measurement based on the first harmonic component offered better detection limits. Therefore, the components at higher frequencies than fundamental frequency were removed from the current response. The filtration was initially done by utilizing analog filters. A series of low pass filters were located before the A/D converter board. However, such filter may cause a small distortion in the magnitude and phase of the fundamental harmonic (which can be determined by calibrating). Also, a digital filtration was occurred during data acquisition. If the excitation potential and the electrode response can be represented by periodic functions, the electrode admittance can be calculated. Briefly in order to avoid problems with the interpretation of the electrode admittance, it is necessary to remove all components of the electrode response at frequencies higher than half the data acquisition frequency. This condition is known as the Nyquist sampling theorem (Weaver, 1983).

The examples of the SW voltammetric responses on the Au UME in the FIA measurement are shown in Figure 6. The analyte signal appears as a current decline in certain potential at the SW voltammogram. It results the inhibition of the electrode surface processes by the analyte adsorption process (Norouzi et al., 2009). To visualize the dependence of the analyte signal to the electrode potential in Figure 6a, the differential form of the SW voltammograms are shown (Figure $6 \mathrm{~b}$ ). In the differential graphs, also, it can be noted that the analyte signal extends over a potential range of the SW voltammogram. 

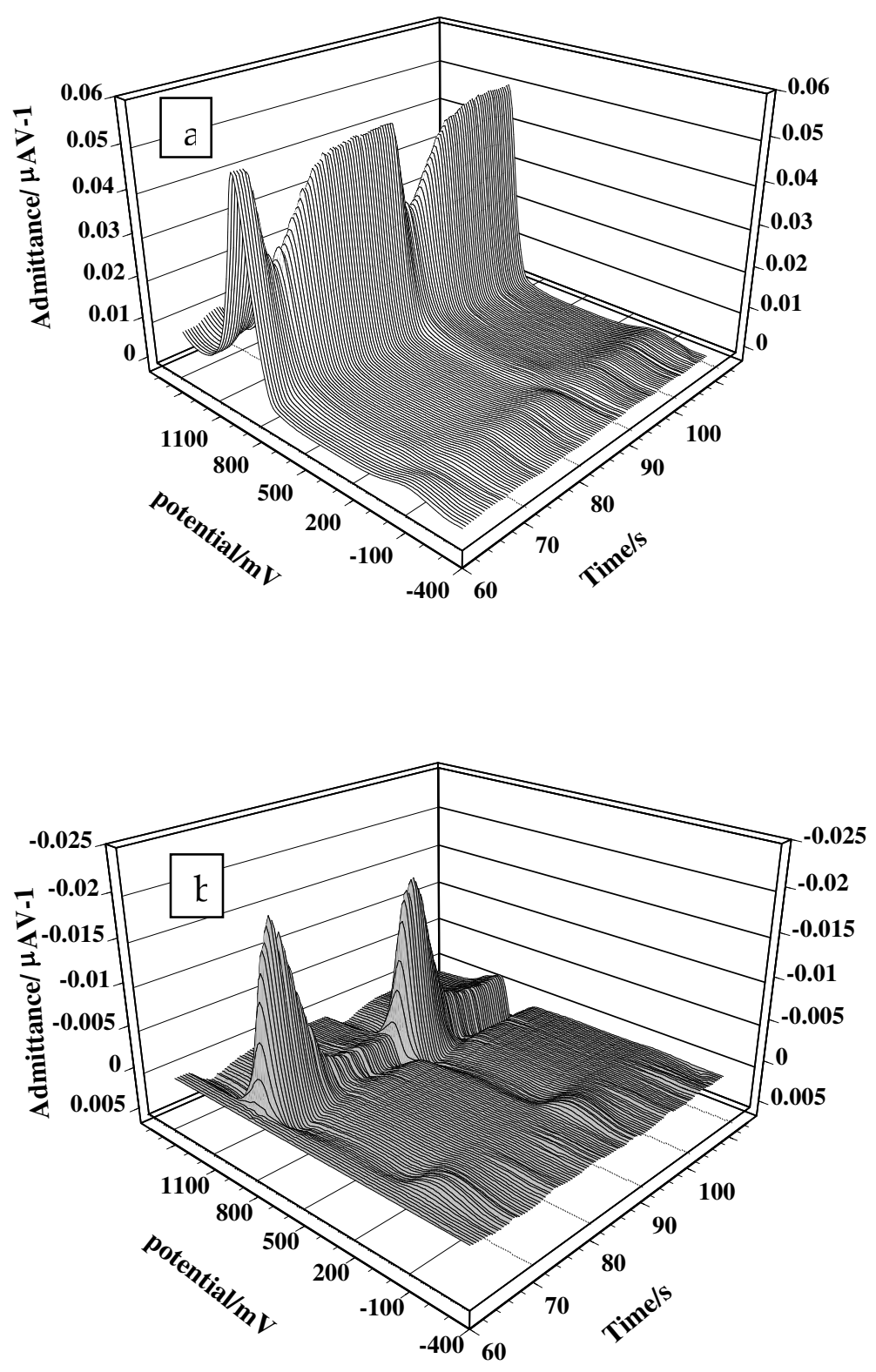

Fig. 6. a) FFT SW voltammograms at Au ultra-microelectrode recorded during the flow injection. The eluent was $0.05 \mathrm{M} \mathrm{H}_{3} \mathrm{PO}_{4}$ with the flow rate of $0.5 \mathrm{~mL} / \mathrm{min}$., b) Curves resulted from subtracting the SWs in fig. a, from the average of 10 SWs (in the absence of analyte) 


\section{Conclusion}

Electroanalytical techniques can offer rapid and low cost analysis of electroactive compounds and heavy metal ions in aqueous systems with a parts-per-billion sensitivity range. Electrical signals may be examined in both the time and in the frequency domain. The two display modes are related to each other by FTT, so each signal variable in the time domain has a characteristic frequency spectrum and vice versa. In the FFT based EC method (such as ENA, FFT Cyclic voltammetry, FFT SW voltammetry and FFT impedance spectroscopy), initially, an electrode response was recorded. Then, FFT was applied on the collected data and the existing high frequency noises were indicated. Based on this information, the cutoff frequency of the analog filter was set at a certain value (where the noises were removed from the electrode response). The smoothing function is, effectively, a moving average filter which is applied to the transfer function data before it is displayed in order to minimize the presence of jagged edges and discontinuities in the displayed data. Finally with the aid of this function a displayed trace can be smoothed by averaging over several electroanalytical measurements. Therefore the signal at the spectrum analyzer input may give rise to unwanted components which do not show any relationship to the input signal.

Some of the major advantages of FFT-voltammetry over other electrochemical techniques include:

- Speed: Because all of the frequencies are measured simultaneously, most measurements by FFT-voltammetry are made in a matter of nano seconds rather than several minutes.

- Sensitivity: Sensitivity is dramatically enhanced with FFT-voltammetry for many reasons. The detectors employed are much more sensitive, the electrical throughput is much higher which results in much lower noise levels, and the fast scans enable the coaddition of several scans in order to reduce the random measurement noise to any desired level (referred to as signal averaging).

Finally, the sensitivity and accuracy of electroanalytical methods based on FFT, along with a wide variety of software algorithms, have dramatically increased the practical use of voltammograms for quantitative analysis. Quantitative methods can be easily developed and calibrated and can also be incorporated into simple procedures for routine analysis. Thus, the FFT-electroanalytical techniques have brought significant practical advantages to other electroanalytical methods. It has made possible the development of many new sampling techniques which were designed to tackle challenging difficulties which were impossible by older technologies. It has made the application of electroanalytical analysis virtually limitless.

\section{References}

Aballe, A., Bethencourt, M., Botana, F.J. \& Marcos, M. (1999). Using wavelets transform in the analysis of electrochemical noise data. Electrochim. Acta, 44, 26, 4805-4816.

Arundell, M., Patel, B.A., Yeoman, M.S., Parker, K.H. \& O'Hare, D. (2004) Hilbert transform of voltammetric data . Elecrochem. Commun., 6, 4, 366-372.

Baranski, A., \& Szulborska, A. (1994) Fourier transform square-wave voltammetry. $d$ Electroanal. Chem., 373, 157-165.

Baranski, A.S., Norouzi, P. \& Nelsson, L.J. (1996). Proc. Electrochem. Soc., 9, 41. 
Bond, A.M., Schwall, R.J. \& Smith, D.E. (1977). On-line FFT faradaic admittance measurements application to A.C. cyclic voltammetry. I Electroanal. Chem., 85, 2, 231-247.

Brett, C.M.A. \& Brett, A.M.O. (1993). Electrochemistry: Principles, Methods, and Applications, Oxford University Press, Oxford.

Carstensen, J., Foca, E., Keipert, S., Foell, H., Leisner M. \& Cojocaru, A. (2008) New modes of FFT impedance spectroscopy applied to semiconductor pore etching and materials characterization . Phys. Status Solidi A, 205, 11, 2485-2503.

Dai, Y. (2000). The time-frequency analysis approach of electric noise based on the wavelet transform. Solid-State Electron., 44, 12, 2147-2153.

Daneshgar, P., Norouzi, P., Ganjali, M.R. \& Zamani, H.A. (2009). Ultrasensitive flowinjection electrochemical method for detection of anticancer drug tamoxifen. Talanta, 77, 3, 1075-1080.

Darowicki, K. \& Zieliski, A. (2001). Joint time-frequency analysis of electrochemical noise. $\mathscr{C}$ Electroanal. Chem., 504, 2, 201-207.

Ganjali, M.R., Norouzi, P., Ghorbani, M. \& Sepehri, A. (2005). Fourier transform cyclic voltammetric technique for monitoring ultratrace amounts of salbutamol at gold ultra microelectrode in flowing solutions. Talanta, 66, 5, 1225-1233.

Garland, J.E., Assiongbon, K.A., Pettit, C.M., Emery, S.B. \& Roy, D. (2002) Kinetic analysis of electrosorption using fast Fourier transform electrochemical impedance spectroscopy: underpotential deposition of $\mathrm{Bi} 3+$ in the presence of coadsorbing ClO4- on gold. Electrochim. Acta, 47, 25, 4113-4124.

Gavaghan, D.J. \& Bond, A.M. (2000). A complete numerical simulation of the techniques of alternating current linear sweep and cyclic voltammetry: analysis of a reversible process by conventional and fast Fourier transform methods. If Electroanal. Chem., 480, 1-2, 133-149.

Házì, J., Elton, D.M., Czerwinski, W.A., Schiewe, J., Vicente-Beckett, V.A. \& Bond, A.M. (1997) Microcomputer-based instrumentation for multi-frequency Fourier transform alternating current (admittance and impedance) voltammetry. $J$ Electroanal. Chem., 437, 1-2, 1-15.

Lafront, A.M., Safizadeh, F., Ghali, E. \& Houlachi, G. (2010) Study of the copper anode passivation by electrochemical noise analysis using spectral and wavelet transforms. Electrochim. Acta, 55, 7, 2505-2512.

Norouzi, P., Ganjali, M.R. \& Meibodi, A.S.E. (2008) A novel adsorptive square wave voltammetric method for pico molar monitoring of lorazepam at gold ultra microelectrode in a flow injection system by application of fast Fourier transform analysis. Anal. Lett., 41, 7, 1208-1224.

Norouzi, P., Ganjali, M.R., Daneshgar, P., Dinarvand, R., Moosavi-Movahedi, A.A. \& Saboury, A.A. (2007). Development of fast Fourier transform continuous cyclic voltammetry at Au microelectrode in flowing solutions as a novel method for subnanomolar monitoring of lidocaine in injection and biological fluids. Anal. Chim. Acta, 590, 1, 74-80.

Norouzi, P., Ganjali, M.R., Nouryousefi, E., Shahtaheri, S.J. \& Dinarvand, R. (2010). Trace Detection of Diphenhydramine by Adsorption on a Microelectrode at Flow Injection System by Fast Fourier Transform Continuous Cyclic Voltammetry . Croatica Chem. Acta, 83, 2, 135-142, b. 
Norouzi, P., Ganjali, M.R., Shahtaheri, S.J., Dinarvand, R. \& Hamzehpoor, A. (2009). Monitoring of Methyldopa by Fast Fourier Transform Continuous Cyclic Voltammetry at Gold Microelectrode .Chinese eI Chem., 27, 4, 732-738.

Norouzi, P., Garakani, T.M., Rashedi, H., Zamani, H.A. \& Ganjali M.R. (2010) Ultrasensitive Flow-Injection Electrochemical Method Using Fast Fourier Transform Square-Wave Voltammetry for Detection of Vitamin B-1. Int. el Electrochem. Sci., 5, 5, 639-652, c.

Norouzi, P., Rashedi, H., Mirzaei Garakani, T., Mirshafian, R., Ganjali, M. R. (2010). Fast Fourier transformation with continuous cyclic voltammetry at a Pt-Au dual microelectrode for the determination of Chloramphenicol in a flow injection system, Int. I Electrochem. Sci., 5, 377 - 39, a.

Osteryoung, J. \& O’Dea, J.J. (1986). Electroanalytical Chemistry, in Bard, A.J., (ed.) Vol. 14, Dekker, New York, p. 209.

Pilla, A.A. (1972). Computers in Chemistry and Instrumentation, in Mattson, J.S., Mark Jr., H.B. \& MacDonald Jr., H.C. (eds.) Vol. 2, Dekker, New York.

Popkirov, G.S. (1996). Fast time-resolved electrochemical impedance spectroscopy for investigations under nonstationary conditions. Electrochem. Acta, 41, 7-8, 1023-1027.

Rauscher, C., Janssen V. \& Minihold R. (2001). Fundamentals of Spectrum Analysis, First edition, Rohde \& Schwarz GmbH \& Co. KG, Mühldorfstrasse 15, 81671 München, Germany.

Safizadeh, F. \& Ghali E. (2010). Monitoring Passivation of Cu-Sb and Cu-Pb Anodes During Electrorefining Employing Electrochemical Noise Analyses. Electrochim. Acta, In Press, Accepted Manuscript, Available online 21 September.

Sang, Y.F., Wang, D., Wu, J.C., Zhu, Q.P. \& Wang, L. (2009). The relation between periods' identification and noises in hydrologic series data.. H Hydrol., 368, 1-4, 30 165-177.

Smith, D.E. (1976) Anal. Chem., 48, 221A-517A.

Van Valkenburg, M.E. (1982). Analog Filter Design, Holt, Rinehart and Winston, New York.

Weaver, H.J. (1983). Applications of Discrete and Continuous Fourier Analysis, Wiley, New York.

Weaver, H.J. (1983). Applications of Discrete and Continuous Fourier Analysis, Wiley, New York.

Winston, S., O’Dea, J.J., \& Osteryoung, J. (1988). Square wave voltammetry for the determination of kinetic parameters: The reduction of zinc(II) at mercury electrodes. el Electroanal. Chem., 255, 21-44.

Zaveri, N., Sun, R., Zufelt, N., Zhou, A. \& Chen, Y.Q. (2007). Evaluation of microbially influenced corrosion with electrochemical noise analysis and signal processing. Electrochim. Acta, 52, 19, 5795-5807. 


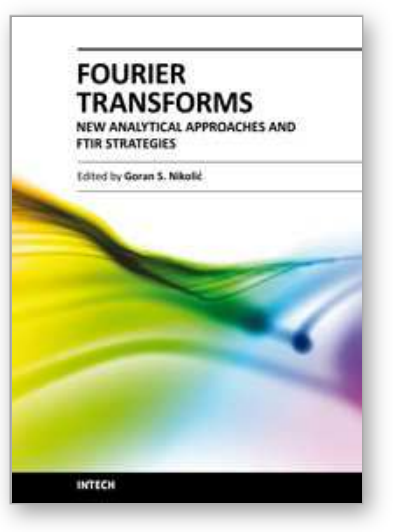

\author{
Fourier Transforms - New Analytical Approaches and FTIR \\ Strategies \\ Edited by Prof. Goran Nikolic
}

ISBN 978-953-307-232-6

Hard cover, 520 pages

Publisher InTech

Published online 01, April, 2011

Published in print edition April, 2011

New analytical strategies and techniques are necessary to meet requirements of modern technologies and new materials. In this sense, this book provides a thorough review of current analytical approaches, industrial practices, and strategies in Fourier transform application.

\title{
How to reference
}

In order to correctly reference this scholarly work, feel free to copy and paste the following:

Parviz Norouzi, Morteza Pirali-Hamedani, Tayebeh Mirzaei Garakani and Mohammad Reza Ganjali (2011). Application of Fast Fourier Transforms in Some Advanced Electroanalytical Methods, Fourier Transforms New Analytical Approaches and FTIR Strategies, Prof. Goran Nikolic (Ed.), ISBN: 978-953-307-232-6, InTech, Available from: http://www.intechopen.com/books/fourier-transforms-new-analytical-approaches-and-ftirstrategies/application-of-fast-fourier-transforms-in-some-advanced-electroanalytical-methods

\section{INTECH}

open science | open minds

\author{
InTech Europe \\ University Campus STeP Ri \\ Slavka Krautzeka 83/A \\ 51000 Rijeka, Croatia \\ Phone: +385 (51) 770447 \\ Fax: +385 (51) 686166 \\ www.intechopen.com
}

\author{
InTech China \\ Unit 405, Office Block, Hotel Equatorial Shanghai \\ No.65, Yan An Road (West), Shanghai, 200040, China \\ 中国上海市延安西路65号上海国际贵都大饭店办公楼405单元 \\ Phone: +86-21-62489820 \\ Fax: +86-21-62489821
}


(C) 2011 The Author(s). Licensee IntechOpen. This chapter is distributed under the terms of the Creative Commons Attribution-NonCommercialShareAlike-3.0 License, which permits use, distribution and reproduction for non-commercial purposes, provided the original is properly cited and derivative works building on this content are distributed under the same license. 